\title{
Terapia ecoguidata delle cisti renali
}

\author{
G. Balconi \\ Servizio di Radiologia Diagnostica. Istituto Scientifico H S. Raffaele - Milano
}

S eppur note da tempo le cisti riscontro con l'avvento della tecnologia ad ultrasuoni. Infatti queste cisti sono nella maggior parte dei casi del tutto asintomatiche e pertanto, prima dell'avvento delle indagini con ultrasuoni, non venivano generalmente diagnosticate. Il riscontro ecografico di cisti renali è per lo più occasionale, in corso di esami effettuati per altre motivazioni.

Le cisti renali interessano prevalentemente la popolazione dopo la quinta decade di vita, mentre appare raro il loro riscontro in età pediatrica. Dopo i 50-60 anni le cisti renali sono presenti in circa il $10 \%$ della popolazione, con una lieve prevalenza per il sesso femminile (1). Vengono ovviamente escluse da questa valutazione le formazioni cistiche renali su base ereditaria.

Per quanto riguarda quindi le cisti renali semplici esse rappresentano un'entità anatomo-patologica ben definita anche se la loro eziopatogenesi è ancora controversa. Le cisti renali semplici possono essere uniche o multiple, uni o bilaterali e, nella maggior parte dei casi, si presentano con cavità singola. Esse presentano all'indagine ecografica, caratteristiche pressoché patognomoniche, costituite da immagine transonica, con pareti nette e regolari e con il classico rinforzo di parte distale. In assenza di renali sono divenute di usuale sintomi e di alterazioni di esami ematochimici la diagnosi ecografica ha un ragionevole grado di certezza e può ritenersi generalmente conclusiva. La valutazione ecografica raggiunge infatti un'accuratezza diagnostica attorno al 90-100\%. Tale diagnosi può essere confermata, con un'accuratezza diagnostica attorno al $100 \%$, dalla TC (2). In presenza di sintomi o quando la raccolta transonica non appare ecograficamente tipi$\mathrm{ca}$, per irregolarità delle sue pareti, per la presenza di multipli setti, magari irregolari, o per la presenza di vegetazioni al suo interno, sono indispensabili ulteriori indagini non essendo l'ecografia sufficientemente sicura nel porre una diagnosi definitiva. Si ricorrerà in questo caso ad esami come la TC ed eventualmente ad agoaspirato a scopo diagnostico. Con le apparecchiature ecografiche attuali la puntura ecoguidata

delle cisti a scopo diagnostico appare comunque sempre più limitata.

In presenza di cisti renali l'atteggiamento terapeutico può essere di nessun trattamento, quando le formazioni cistiche sono di piccole dimensioni e asintomatiche oppure comportare una terapia di elezione, di asportazione della cisti, qualora questa si presenti sintomatica. Solo in rarissimi casi di torsione della cisti o di rottura della stessa o di sanguinamento intra-cistico può essere indicata una terapia chirurgica d'urgenza.

La terapia d'elezione delle cisti renali può essere effettuata con intervento chirurgico a cielo aperto oppure con intervento in laparoscopia.

E possibile effettuare lo svuotamento della cisti renale anche con manovre percutanee, generalmente sotto guida ecografica (Tab. I). Ci occuperemo di questa ultima metodica.
TAB. I - POSSIBILI TERAPIE DELLE CISTI RENALI

- Intervento chirurgico

- Agoaspirazione

- Agoaspirazione con iniezioni sclerosanti

- Asportazione per via laparoscopica 
TAB. II - INDICAZIONI ALLA TERAPIA DELLE CISTI RENALI

- Compressione strutture del seno pielico

- Stasi urinaria

- Ostacolo al flusso vascolare

- Sintomi correlabili con la presenza della cisti

- Lombalgia

- Coliche

- Sospetta ipertensione nefro-vascolare

- Rapido incremento delle dimensioni

- Effetto massa

\section{Terapia delle cisti renali: quando?}

Le indicazioni alla terapia delle cisti renali sono rappresentate fondamentalmente da quattro motivazioni: la compressione su struttura del seno pielico, la presenza di sintomi correlabili alla cisti, il rapido incremento delle dimensioni della cisti e l'effetto massa $(3,4)$ (Tab. II).

La compressione delle strutture del seno pielico può comportare stasi urinaria, di tutti i distretti caliciali e del bacinetto stesso, per compressione sul tratto prossimale dell'uretere o segmentaria, di alcuni calici. La compressione sul seno pielico può determinare anche ostacolo al flusso vascolare, sia arterioso, con possibile comparsa di ipertensione, sia venoso, con sintomi da stasi dei distretti eventualmente interessati (ad esempio varicocele sinistro per compressione della vena spermatica prima del suo ingresso nella vena renale).

I sintomi correlabili con la presenza della cisti sono riconducibili a lombalgie, a coliche o a ipertensione nefro-vascolare. Purtroppo in tali casi non abbiamo la possibilità di documentare con certezza che la sintomatologia sia riferibile alla formazione cistica. Solo la sua puntura evacuativa, con la relativa scomparsa della sintomatologia ne permette quindi un accertamento.

Il rapido incremento delle dimensioni di una cisti può rappresentare una buona indicazione all'intervento di elezione sulla sua asportazione o evacuazione in quanto è prevedibile che a distanza di poco tempo possono comparire sintomatologie connesse alla cisti stessa.
Per effetto massa si intende l'impronta su visceri quali intestino, ureteri, vie biliari extra epatiche o su strutture vascolari circostanti, oppure la dislocazione del rene rispetto ad organi vicini.

La sintomatologia legata alle cisti renali si presenta comunque quando queste raggiungono dimensioni relativamente cospicue, generalmente superiori ai $10 \mathrm{~cm}$ di diametro (5).

La comparsa di ematuria, in presenza di cisti renali, non appare invece, alla luce dei dati attuali (6), correlabile alla presenza di formazioni cistiche. Pertanto il verificarsi di un'ematuria, in concomitanza con la presenza di una cisti, non può essere considerata un'indicazione alla aspirazione o all'asportazione della cisti stessa. Le motivazioni dell'ematuria vanno quindi ulteriormente indagate e non etichettate semplicemente come dipendenti dalla presenza della cisti.

Non viene invece ritenuta una indicazione alla asportazione o alla evacuazione di una cisti renali la presenza di reni policistici. Non è mai stato infatti dimostrato che riducendo il volume delle cisti o i loro numero sia possibile rallentare la progressione dei danni renali nei reni policistici.

\section{Terapia percutanea delle cisti renali: come?}

Accertata l'indicazione all'intervento per una cisti renale è possibile la sua asportazione chirugica o il suo svuotamento percutaneo. La tecnica dello svuotamen- to percutaneo sembra attualmente la procedura più sicura e meno traumatica.

\section{Apparecchiature di guida}

I primi trattamenti percutanei delle cisti renali risalgono al 1986 (7). Queste prime esperienze venivano condotte sotto guida fluoroscopica ed era pertanto necessaria una sala di radiodiagnostica, con amplificatore di brillanza. Il controllo fluoroscopico consente il vantaggio di introdurre del mezzo di contrasto nella cavità cistica e pertanto di evidenziarne una eventuale sua connessione con altre strutture. Presenta invece lo svantaggio di una puntura non strettamente monitorata e soprattutto può comportare una certa incidenza di recidive e complicanze in quanto è sempre possibile la dislocazione della punta dell'ago, specialmente alla fine dell'aspirazione quando la membrana della cisti è ormai collassata. La dislocazione della punta dell'ago, a cisti collassata dopo il suo svuotamento, può determinare gravi complicanze alla successiva introduzione dei liquidi sclerosanti che vengono iniettati non nelIa cavità cistica ma in strutture circostanti. Anche la metodica TC, seppur più precisa rispetto alla metodica fluoroscopica, può comportare le stesse complicanze legate alla dislocazione della punta dell'ago dopo evacuazione della cisti stessa.

La guida ecografica consente invece un monitoraggio costante e continuo sia della puntura della cisti, sia del suo svuotamento, sia nelle successive manovre di sclerotizzazione.

La procedura più corretta potrebbe essere quella di effettuare la manovra su di un tavolo radiologico, monitorando la puntura della cisti sotto guida ecografica, effettuando un controllo fluoroscopico dopo introduzione di mezzo di contrasto, e continuando il monitoraggio ecoguidato nelle fasi di sclerotizzazione.

Per quanto riguarda l'apparecchiatura ecografica è necessario disporre di apparecchiatura in tempo reale, con sonde di 3.5-5 MHz, settoriali o convex, che meglio di adattano alla manovra interventistica. Possono essere utilizzate sonde dedicate per ecografia interventistica, adattatori guida aghi, applicabili a tutte le sonde oppure è possibile procedere alla manovra a mano libera: l'ago è infatti 


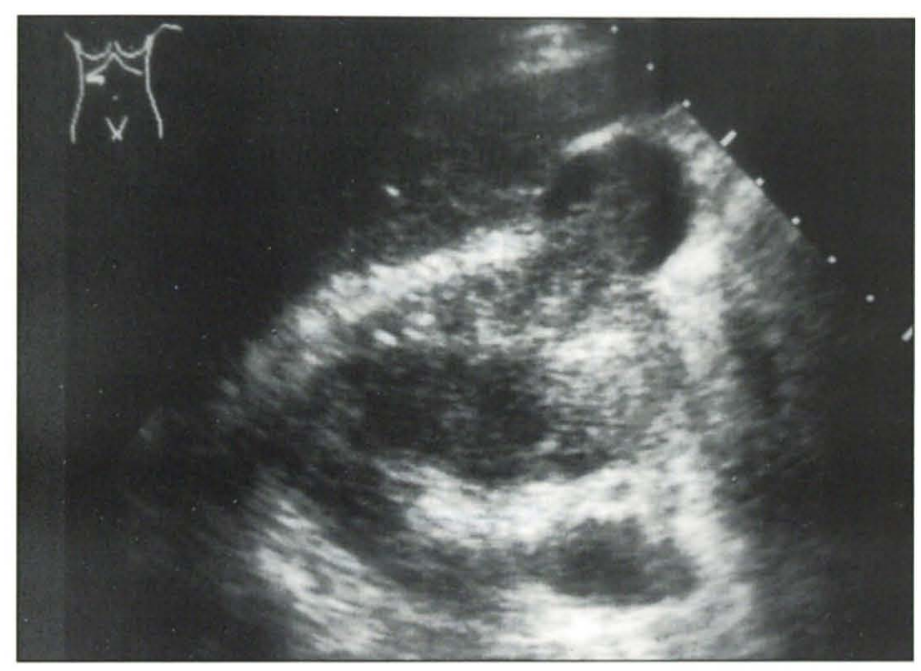

Fig. 1 - All' interno di formazioni cistiche renali sono possibili anche fenomeni emorragici spontanei, con formazione di trombi adesi alle pareti, che possono ingenerare dubbi diagnostici. Come nell' esempio riportato, all' interno della formazione cistica (1) si evidenzia un trombo ematico (frecce), adeso alla parete della cisti. In questi casi è opportuno, prima di procedere all' alcoolizzazione, l'esame del liquido aspirato onde escludere ipotesi neoplastiche.

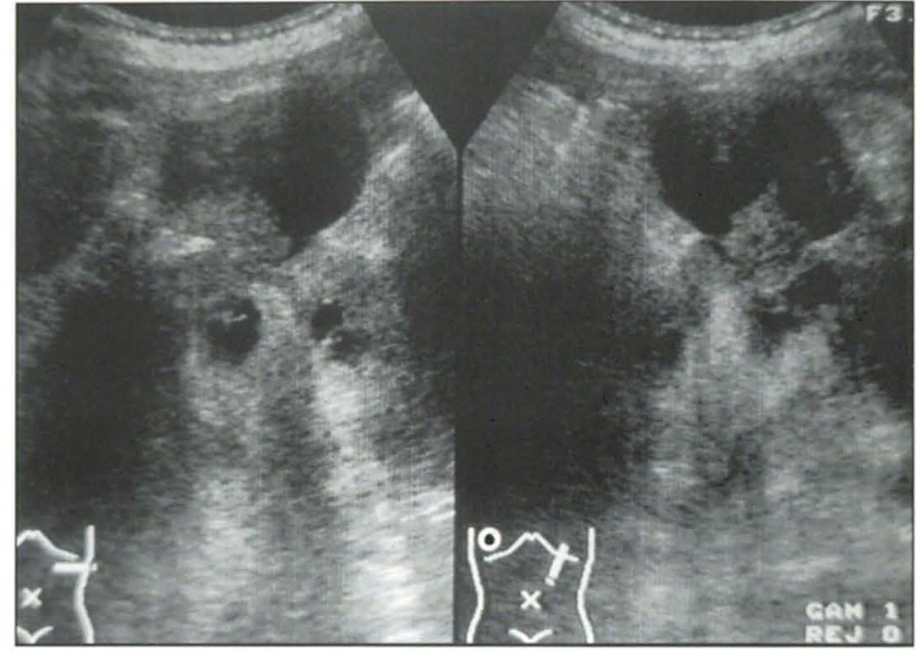

Fig. 2 - In A scansione assiale del rene sinistro e in B scansione longitudinale del rene sinistro. Sul margine laterale del rene è presente formazione cistica (1) nel cui interno si rileva la presenza di formazione vegetante (frecce). L'ago aspirazione ecoguidata ha permesso di evidenziare la presenza di cellule neoplastiche. ben riconoscibile all'ecoscopia e pertanto è possibile procedere con un costante controllo dello stesso. È indubbio che le sonde dedicate oppure dispositivi guida aghi possono rendere più agevole e più automatica la manovra stessa.

\section{Puntura ecoguidata delle cisti renali: come si esegue}

In presenza di criteri indicativi per una terapia della cisti renale è possibile effettuare lo svuotamento della stessa con agoaspirazione oppure svuotamento della cisti con successiva procedura di sclerotizzazione delle pareti della stessa.

La puntura evacuativa può essere effettuata con ago sottile, atraumatico, sotto guida ecografica. Lo svuotamento della cisti con agoaspirazione si è dimostrata una metodica estremamente agevole, sicura e utile soprattutto nella fase diagnostica, in caso di dubbi sulla natura della cisti stessa (Figg. 1, 2), mentre da un punto di vista terapeutico tale procedura non riscontra un significativo successo. Infatti le recidive dopo evacuazione delle cisti appaiono estremamente elevate $(2,8,9)$.

Se pertanto si vuole ottenere una buona risposta terapeutica bisogna cercare di creare un collabimento stabile delle pareti della cisti. Si è ottenuto tale risultato introducendo nella cisti stessa delle sostanze sclerosanti.

In passato sono state utilizzate diverse sostanze sclerosanti, le più importanti delle quali sono riportate nella Tabella III.

Dopo le prime esperienze con alcool etilico $95^{\circ}$ effettuate nel 1976, l'utilizzo di tale sostanza è divenuto di scelta a partire dal 1981 (8). La scelta di tale sostanza è dettata dal suo basso costo e dalla sua elevata maneggevolezza. Nel 1989 Holmberg e Hietala hanno proposto l'uso del bismuto fosfato, con risultati che appaiono assai promettenti, ma ancora da codificare in modo definitivo (9).

TAB. III - TERAPIA PERCUTANEA DELLE CISTI RENALI: SOSTANZE SCLE-

\section{Procedura di alcolizzazione ecoguidata delle cisti renali}

È opportuna l'esecuzione della manovra in ambiente ospedaliero, anche se non in regime di ricovero.

È corretto il consenso informato del paziente, il controllo della sua coagulazione, il digiuno da almeno 6 ore. Ė opportuno effettuare la manovra su un tavolo radiologico in modo da poter disporre sia della guida ecografica sia eventualmente del controllo fluoroscopico.

\section{ROSANTI UTILIZZATE}

\begin{tabular}{|l|}
\hline - Glucosio \\
- Fenolo \\
- Iofendilato \\
- Urea cloidrolattato \\
- Pantopaque \\
- Tetracicline \\
- Fibrina umana
\end{tabular}

- Alcool etilico sterile $\mathbf{9 5}^{\circ}$

(Bean 1981)

- Bismuto fosfato

(Holmberg 1989) 
Il paziente viene posizionato in decubito prono, eventualmente con sollevamento parziale dell'addome, ponendo un cuscino al di sotto dell'addome del paziente.

Viene preparato campo sterile con disinfezione cutanea secondo le usuali metodiche chirurgiche. Viene adeguatamente prescelto il tragitto da percorrere con l'ago, nei limiti del possibile scegliendo un tragitto sottocostale, subito a lato della muscolatura paravertebrale. In tale sede lo spessore della parete muscolare è infatti modesto e si è certi di rimanere nello spazio retroperitoneale. Può essere utilizzato, se indispensabile e con maggiori cautele, anche lo spazio intercostale, come descritto da alcuni Autori che hanno effettuato punture di cisti renali TC guidate (10).

$\mathrm{Si}$ effettua quindi anestesia locale con Xilocaina $2 \%$. L'anestesia locale deve interessare cute, sottocute e lo strato muscolare. Si effettua poi incisione con bisturi a punta per una profondità di circa $1.5-2 \mathrm{~cm}$.

Per l'alcolizzazione delle cisti renali si ritiene ormai comunemente opportuno posizionare un piccolo catetere all'interno della cisti attraverso il quale effettuare lo svuotamento della cisti stessa e l'introduzione del mezzo sclerosante. È quasi del tutto abbandonata la procedura effettuata solo con ago in quanto il collabimento delle pareti della cisti, dopo il suo svuotamento, comporta il rischio della fuoriuscita della punta dell'ago dalla cavità cistica. È possibile posizionare un piccolo catetere all'interno della cisti seguendo la metodica di Seldinger oppure con metodiche più rapide, che fondamentalmente utilizzano un catetere con all'interno il mandrino che viene inserito direttamente nella cavità cistica. Sicuramente queste metodiche sono più rapide, ma devono essere utilizzate solo da mani esperte. La metodica di Seldinger, costituita da plurimi passaggi, è sicuramente più indaginosa. La metodica consente comunque di pungere la formazione cistica con aghi relativamente sottili, quindi di ridurre le possibili complicanze da mancata centratura della lesione. Seguiremo pertanto questo tipo di procedura, sicuramente utilizzabile anche da operatori non particolarmente esperti (Tab. IV).

Attraverso l'incisura cutanea effettuata con bisturi a punta si introduce ago di 18 $\mathrm{G}$ che, sotto guida ecografica, viene fatto proseguire fino all'interno della cisti.

TAB. IV - ALCOOLIZZAZIONE DI CISTI RENALE: MODALITÀ DI ESECUZIONE

\begin{tabular}{|l} 
Prima parte \\
\begin{tabular}{|l|} 
- Posizionamento di catetere \\
- tipo Seldinger \\
- tipo Trocar \\
- Svuotamento della cisti \\
- esame biochimico \\
- esame citologico \\
- Esame contrastografico
\end{tabular}
\end{tabular}

Seconda parte
- Introduzione di lidocaina transcatetere

- Introduzione di alcool etilico

- 1/4 - 1/2 del volume della cisti $-<300 \mathrm{ml}$

- Rotazione del paziente

- Aspirazione dell'alcool

- Rimozione del catetere

- Controllo dopo $1 \mathrm{~h}$

A

B

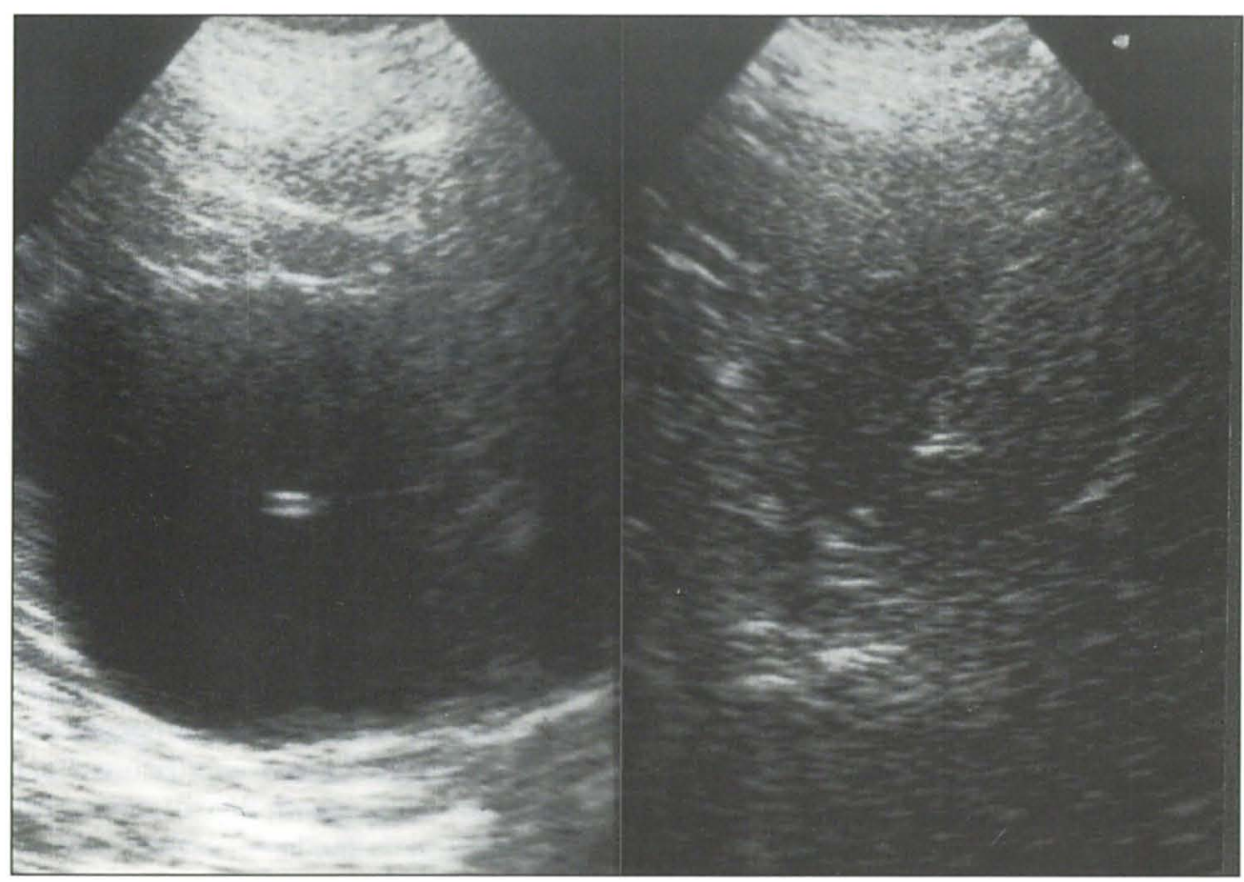

Fig. 3 - In A è riconoscibile il piccolo catetere di drenaggio (freccia) all' interno della formazione cistica (1). Dopo aspirazione completa del liquido è ancora riconoscibile (in $B$ ) il piccolo catetere di drenaggio senza liquido attorno ad esso.

Giunti all'interno della cisti si può controllare, tramite aspirazione attraverso l'ago, la fuoriuscita del liquido cistico. Campione del liquido cistico, oltre ad essere visualizzato macroscopicamente, può essere utilizzato anche per eventuali esami biochimici, batteriologici o citologici (11).

$\mathrm{Si}$ introduce a questo punto una modica quantità di mezzo di contrasto onde escludere eventuali collegamenti della cisti con altre strutture. Il controllo fluoroscopico risulta, a questo punto, di estrema utilità.

$\mathrm{Si}$ introduce quindi attraverso la camicia dell'ago, guida metallica di Seldinger che viene fatta arrotolare, nella sua parte terminale, all'interno della formazione cistica. Si estrae quindi la camicia dell'ago e sulla guida metallica si introduce un piccolo catetere, del diametro massimo di 5-6 F. Si estrae la guida e si aspira at- 
TAB. V - COMPLICANZE DELL'ALCOOLIZZAZIONE DELLE CISTI RENALI

\begin{tabular}{|l|}
\hline - Maggiori \\
- emorragie perirenali \\
- urinomi \\
- pseudoaneurismi \\
- fistole artero-venose \\
- infezioni \\
- pneumotoraci \\
- perforazioni di organi vicini
\end{tabular}

$$
\begin{aligned}
& \text { Minori } \\
& \text { - dolore locale } \\
& \text { - febbre } \\
& \text { - nausea - vomito } \\
& \text { - coliche } \\
& \text { - ematuria }
\end{aligned}
$$

- emorragia intracistica

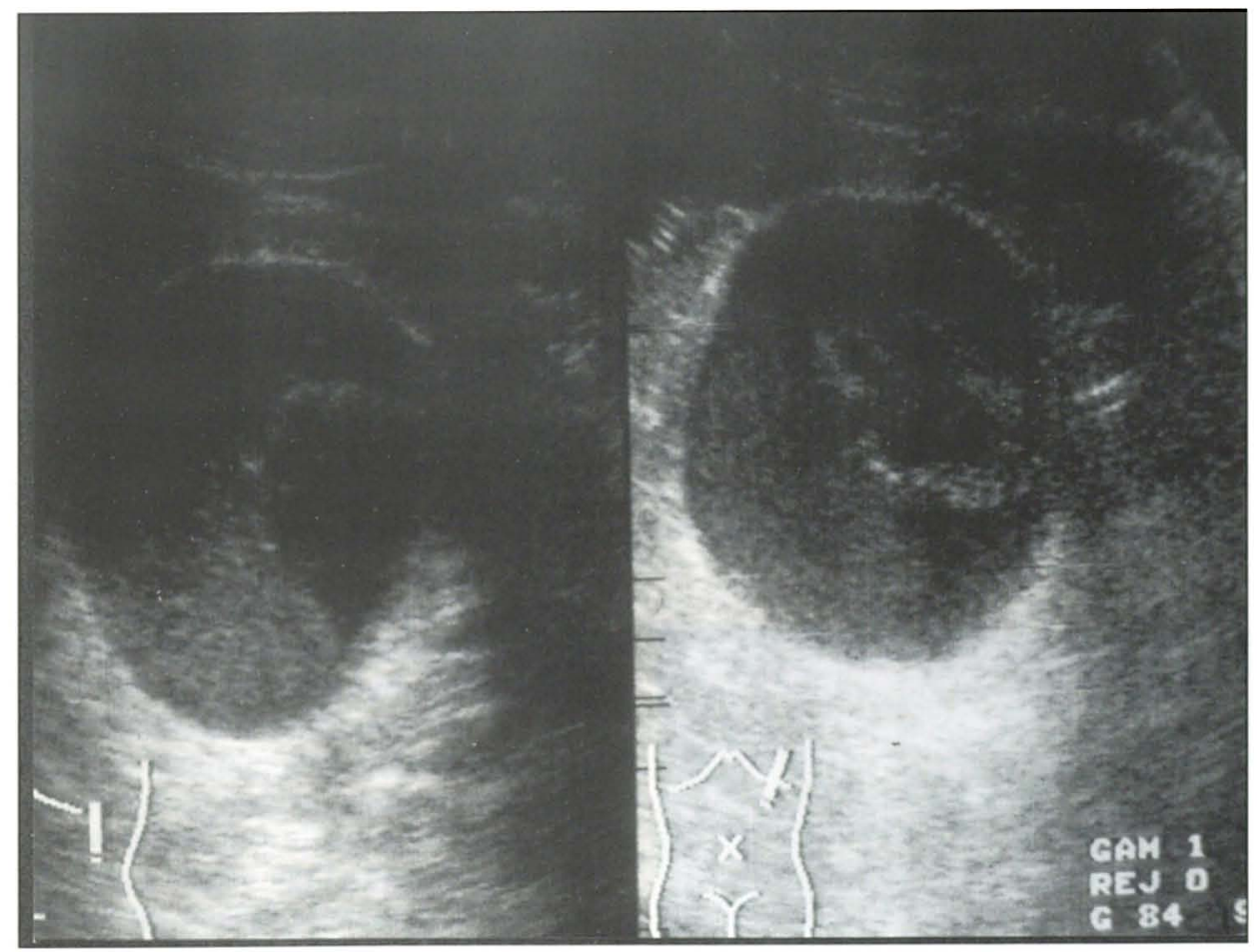

Fig. 4 - Alcoolizzazione percutanea di cisti renale (1). Dopo svuotamento della cisti attraverso un piccolo catetere (frecce) si introduce alcool etilico $95^{\circ}$. L'alcool può apparire francamente iperecogeno al momento della sua introduzione nella cisti.

traverso il catetere il liquido cistico fino al completo svuotamento della cisti stessa (Fig. 3).

Alcuni Autori (5) ritengono opportuno introdurre pochi cc di lidocaina all'interno della cisti prima dell'introduzione dell'alcool. Secondo altri Autori questa procedura non è necessaria.

$\mathrm{Si}$ introduce quindi alcool etilico $95^{\circ}$, sterile, attraverso il catetere, nella cavità cistica in quantità pari a circa $1 / 3$ del vo- lume della cisti (controllabile attraverso la quantità di liquido aspirato) all'interno della cisti (Fig. 4). Il volume complessivo di alcool da introdurre non deve superare, secondo alcuni Autori, anche per le cisti più voluminose, i $100 \mathrm{cc}$ di alcool (1), mentre secondo altri il volume massimo da introdurre non deve superare $\mathrm{i}$ 300 cc di alcool (5).

L'alcool viene lasciato in situ per circa 30 minuti. Quasi tutti gli Autori sono concordi nel variare il decubito del paziente durante la permanenza dell'alcool all'interno della cisti, per permettere un più uniforme contatto dell'alcool sulle pareti stesse. Tale procedura non è a ritenersi comunque di particolare importanza in quanto, se si è avuta l'accortezza di non introdurre dell'aria nelle varie fasi del procedimento di svuotamento della cisti, all'interno della stessa e non si è lasciato del liquido residuo, il contatto dell'alcool sulle pareti della cisti è costante. Dopo circa 30 minuti viene aspirato completamente l'alcool, rimosso il catetere ed effettuata una semplice medicazione cutanea. È opportuno trattenere il paziente per alcune ore in controllo. In assenza di complicanze dopo alcune ore il paziente può essere rinviato a domicilio.

In alcuni casi, specie in cisti molto voluminose, può essere opportuno, dopo l'alcolizzazione lasciare in situ il piccolo catetere per alcuni giorni. A distanza di alcuni giorni è possibile, tramite lo stesso catetere effettuare una seconda alcoolizzazione.

È stato dimostrato, con controllo dell'alcoolemia, che non vi è passaggio di alcool in circolo (5).

\section{Possibili complicanze dell'alcoolizzazione delle cisti renali}

Per quanto riguarda le complicanze dell'alcoolizzazione delle cisti i dati riportati in letteratura appaiono molto soddisfacenti. Complicanze gravi con mortalità del paziente sono risultate nulle. Le complicanze descritte in letteratura sono distinguibili in maggiori e minori. Le complicanze maggiori comprendono emorragie perirenali, urinomi, pseudoaneurismi, fistole artero-venose, infezioni, pneumotoraci e perforazioni di organi vicini (Tab. V).

Tutte queste complicanze, eccetto le fistole artero-venose, sono legate a punture di strutture esterne, seppur vicine, alla cisti. Tali complicanze erano relativamente frequenti effettuando l'alcoolizzazione sotto guida fluoroscopica. Come già accennato, infatti, lo svuotamento della cisti comportava facilmente la fuoriuscita della punta dell'ago dalla cavità della cisti stessa e la successiva introdu- 


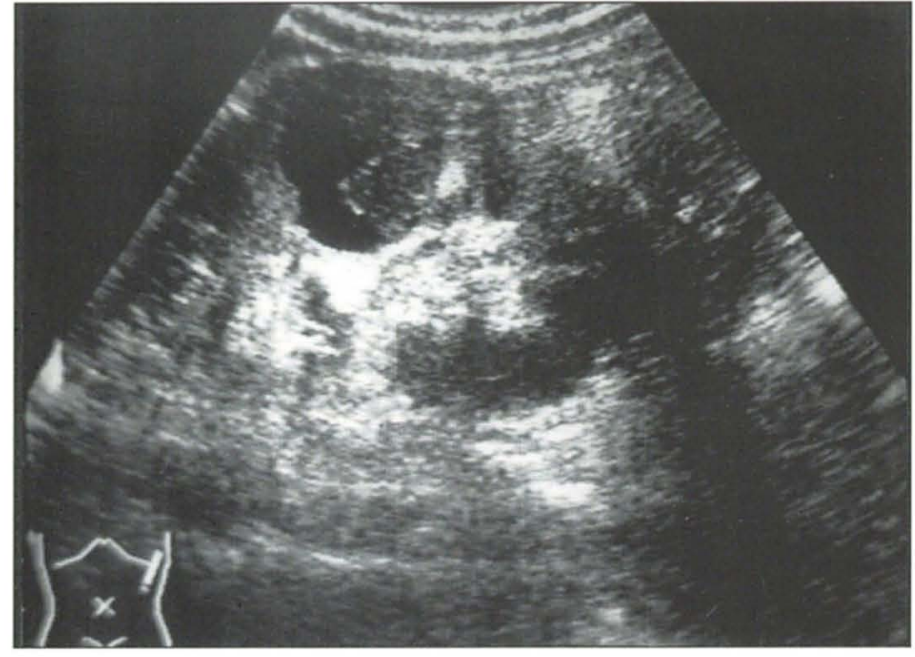

Fig. 5 - Emorragia intracistica. Nel corso della puntura di cisti renale sinistra si è avuta una rapida comparsa, subito dopo il suo svuotamento, di fini echi al suo interno che si sono progressivamente organizzati in una massa iperecogena, riferibile a coagulo ematico. Fra il coagulo ematico (1) e una parte della parete della cisti (frecce) si riconosce una banda ipoecogena costituita da siero.

zione di alcool o di sostanze sclerosanti era causa del danno delle strutture vicine, ove il mezzo sclerosante veniva in realtà iniettato. Tali complicanze sono pressoché inesistenti se la manovra viene effettuata sotto monitoraggio ecografico.

Per quanto riguarda invece le fistole artero-venose tali alterazioni sono riscontrabili anche con puntura ecoguidata ma solo qualora si passi attraverso parenchima renale per giungere all'interno della cavità cistica. Quando possibile la cisti deve essere pertanto punta direttamente,

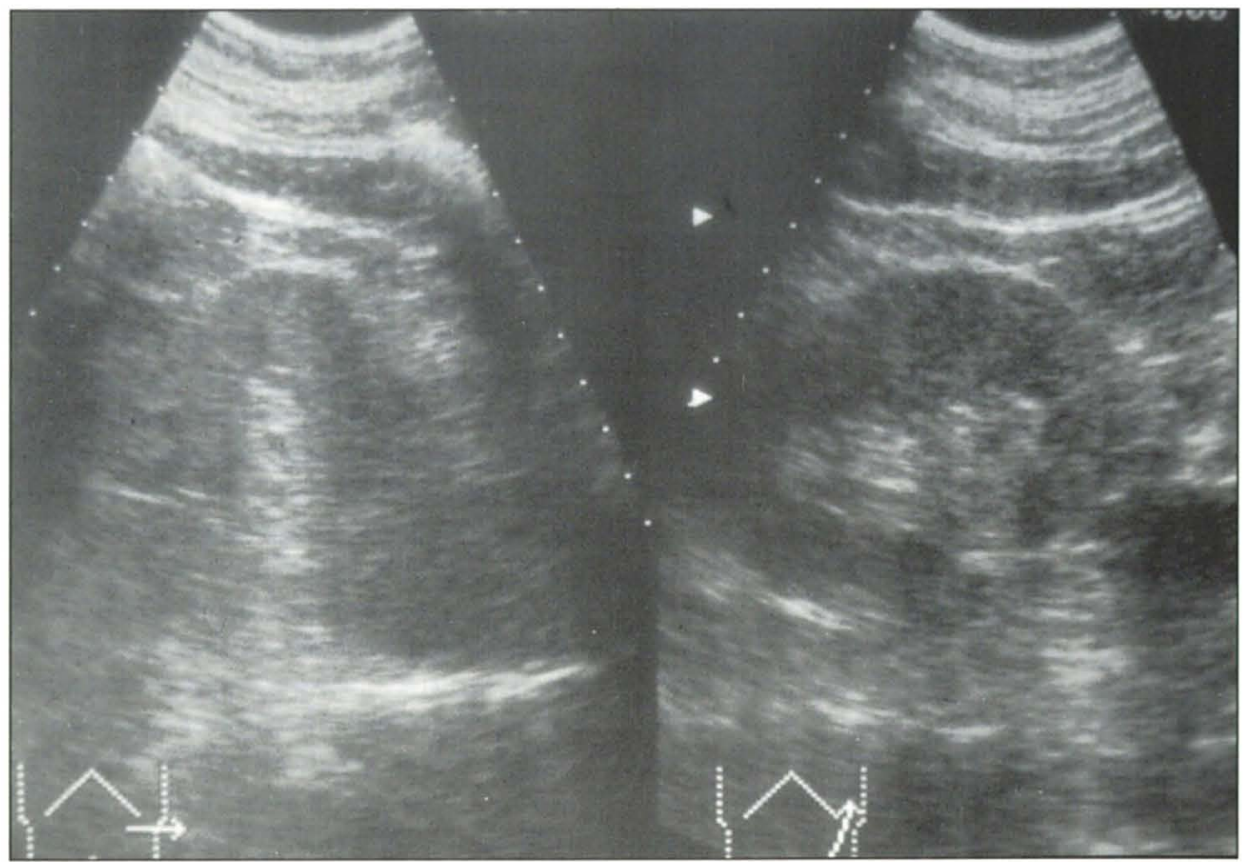

Fig. 6 - Stesso caso della Figg. 3, 4. Controllo a distanza di tre mesi. In A scansione assiale e in B scansione longitudinale del rene sinistro in cui non è più riconoscibile alcuna formazone cistica. non interponendo parenchima renale normale fra la cisti e il percorso dell'ago.

Nelle complicanze minori, di relativo frequente riscontro, si può avere dolore locale, comparsa di febbre modesta, tavolta di nausea e vomito, di sintomatologia tipo colica renale e talvolta anche di modesta ematuria. Tali complicanze minori si risolvono comunque generalmente in modo spontaneo nel corso di poche ore.

Le complicanze documentate risultano comunque inferiori al 5-6\% dei casi trattati (5).

Come si è già detto le complicanze maggiori, segnalate con trattamenti effettuati sotto guida fluoroscopica o sotto guida $\mathrm{TAC}$, non sono mai state segnalate in trattamenti effettuati sotto guida ecografica.

È opportuno un breve richiamo per quanto riguarda l'emorragia intra-cistica. La complicanza appare immediatamente documentabile sia alla ecoscopia (Fig. 5), sia dal fatto che mentre si aspira il liquido cistico, si estrae materiale francamente ematico. La complicanza è priva di importanza in quanto l'emorragia si autolimita. È opportuno in questi casi non continuare ad aspirare il sangue. Non si ritiene comunque prudente proseguire nella manovra di alcoolizzazione al verificarsi di un'emorragia intra-cistica. Molte volte il sangue stesso può determinare delle aderenze, nella sua fase organizzativa, fra le pareti cistiche, determinando la guarigione della cisti stessa. Qualora ciò non si verificasse è tuttavia possibile procedere ad alcoolizzazione a distanza di alcuni giorni. 


\section{Risultati dell'acoolizzazione ecoguidata delle cisti renali}

I dati della letteratura circa i risultati dell'alcoolizzazione delle cisti renali appaiono alquanto discordi (Tab. VI). Bean nel 1981, su Radiology, riferiva di una recidiva di cisti renale su 34 cisti trattate (8). Secondo altri Autori (9) si hanno recidive di cisti renali nel $66 \%$ dei casi, dopo alcoolizzazione delle stesse.

Veltri et al segnalano, nel 1993, la recidiva di cisti renali alcoolizzate in una percentuale dell' $84 \%$ dei casi (5). La spiegazione di tali discrepanze va ricercata nel significato che si vuol dare al successo del trattamento delle cisti renali. Se infatti si ritiene valido un successo legato a una netta riduzione della formazione cistica, in modo permanente, al di sotto dei $5 \mathrm{~cm}$, in modo tale che non possa più essere sintomatica, anche le casistiche meno ottimistiche ottengono un risultato elevato. Nella casistica prima citata di Veltri, infatti le recidive inferiori o uguali a 5 $\mathrm{cm}$, quindi non più sintomatiche, risultano pari ad oltre il $70 \%$. Se invece attribuiamo un successo completo del trattamento alla scomparsa definitiva della cisti (Fig. 6) il risultato che si ottiene è nettamente inferiore.

Restano comunque concordi tutti gli Autori nel ritenere valido un trattamento che comporti la scomparsa o comunque la riduzione della formazione cistica in modo tale che questa non sia più sintomatica. Mantenendo una valutazione di questo tipo il successo complessivo è pari a circa $1^{\prime} 80 \%$. Se nei casi di recidiva viene di nuovo praticato un ciclo di alcoolizzazione le recidive che permangono sono trascurabili, essendo valutate attorno al 2-3\% (1). $\mathrm{Va}$ infine ricordato che molte volte la formazione cistica, recidivata, può tendere nel tempo anche ad una progressiva riduzione del suo volume, spontaneamente. La valutazione complessiva deve pertanto essere effettuata a distanza di alcuni mesi dal trattamento di alcoolizzazione.

Considerato quindi il successo ottenibile nel trattamento ecoguidato percu- taneo con alcool delle cisti renali e la pressoché completa innocuità del trattamento, la metodica potrebbe rappresentare la terapia di elezione del trattamento delle cisti sintomatiche dei reni.

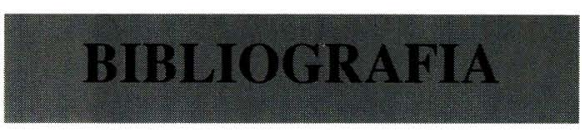

1.

Filice C, Brunetti E, Caremani M. Terapia delle formazioni cistiche. In: Trattato Italiano di Ecografia. Milano: Poletto Edizioni, 1993; 1006.

2. Sandler CM, Houston GK, Hall JT. Guided cyst puncture and aspiration. Radiol Clin North Am 1986; 24: 527-37.

3. Amis ES, Croman JJ, Pfister RC. Needle puncture of cystic renal masses: a survey of the society of uroradiology. AJR 1987; 18: 297-9.

4. Bazzocchi M, Stacul F, Zuliani C. Drenaggi addominali ecoguidati. Radiol Med 1987; 73: 346-52.

5.

Veltri A, Biselli S, Farinet S, Mancini A. Alcolizzazione percutanea ecoguidata delle cisti renali semplici. Radiol Med 1993; 86: 870-5.

6. Bonomo L, Ghimenti A, Picardi N. Ricerca clinica sulla possibilità che le cisti renali siano responsabili della comparsa di ematuria. Ann Ital Chir 1991; 62: 165-70.

7. Raskin MM, Poole DO, Roen SA, Viamonte M. Percutaneous management of renal cysts; results of a four year study. Radiology 1976; 115: 551-3.

8. Bean WJ. Renal cysts: treatment with alcohol. Radiology 1981; 138: 329-31.

9. Holmberg G, Hietala S. Treatment of simple renal cysts by percutaneous puncture and instillation of bismuth-phosphate. Scand J Nephrol 1989; 23: 207-5.
10. Hopper KD, Yakes WF. The posterior intercostal approach for percutaneous renal procedures: risk of punturing the lung, spleen, and liver as determined by CT. AJR 1990; 154: 115-7.

11. Ohkawa M, Motoi I, Hirano S, Okaso A, Hisazumi H. Biochemical analysis and pharmacodynamic studies of simple renal cyst fluid in relation to infection. Nefron 1991; 59: 80 . 